\title{
Is Physical Activity Able to Modify Oxidative Damage in Cardiovascular Aging?
}

\author{
Graziamaria Corbi, ${ }^{1}$ Valeria Conti, ${ }^{2}$ Giusy Russomanno, ${ }^{2}$ Giuseppe Rengo, ${ }^{3}$ \\ Piergiusto Vitulli, ${ }^{1}$ Anna Linda Ciccarelli, ${ }^{1}$ \\ Amelia Filippelli, ${ }^{2}$ and Nicola Ferrara ${ }^{1,3}$ \\ ${ }^{1}$ Department of Medicine and Health Sciences, University of Molise, Via Giovanni Paolo II, Località Tappino, \\ 86100 Campobasso, Italy \\ ${ }^{2}$ Department of Medicine and Sugery, University of Salerno, Via Salvador Allende, 84081 Baronissi (SA), Italy \\ ${ }^{3}$ Istituto Scientifico di Campoli/Telese, Fondazione Salvatore Maugeri, IRCCS, Via Bagni Vecchi 1, 82037 Telese Terme (BN), Italy
}

Correspondence should be addressed to Graziamaria Corbi, graziamaria.corbi@unimol.it

Received 22 June 2012; Accepted 13 August 2012

Academic Editor: William C. Burhans

Copyright (C) 2012 Graziamaria Corbi et al. This is an open access article distributed under the Creative Commons Attribution License, which permits unrestricted use, distribution, and reproduction in any medium, provided the original work is properly cited.

\begin{abstract}
Aging is a multifactorial process resulting in damage of molecules, cells, and tissues. It has been demonstrated that the expression and activity of antioxidant systems (SOD, HSPs) are modified in aging, with reduced cell ability to counteract the oxidant molecules, and consequent weak resistance to ROS accumulation. An important mechanism involved is represented by sirtuins, the activity of which is reduced by aging. Physical activity increases the expression and the activity of antioxidant enzymes, with consequent reduction of ROS. Positive effects of physical exercise in terms of antioxidant activity could be ascribable to a greater expression and activity of SOD enzymes, HSPs and SIRT1 activity. The antioxidant effects could increase, decrease, or not change in relation to the exercise protocol. Therefore, some authors by using a new approach based on the in vivo/vitro technique demonstrated that the highest survival and proliferation and the lowest senescence were obtained by performing an aerobic training. Therefore, the in vivo/vitro technique described could represent a good tool to better understand how the exercise training mediates its effects on aging-related diseases, as elderly with heart failure that represents a special population in which the exercise plays an important role in the improvement of cardiovascular function, quality of life, and survival.
\end{abstract}

\section{Aging and Oxidative Stress}

Aging is a multifactorial process resulting in damage of molecules, cells, and tissues, leading to a reduced efficacy of functions with different pathophysiological consequences and a variety of clinical phenotypes. It is characterized by altered regulation of many genes implicated in stress resistance and processes of tissue regeneration and repair. In particular, old animals are intrinsically less resistant to oxidative stress $[1,2]$.

Several studies have shown that advanced age is associated with abnormalities in cardiac performance and structure, such as a decline in early diastolic left ventricular filling [3] and increases in wall thickness, but also by changes in vascular and cardiac beta-receptor function [4]. Abete et al. [5] demonstrated that changes in response to stress, induced by intracoronary infusion of hydrogen peroxide were pronounced in old rats. In fact they found that cardiac release of oxidized glutathione (an index of the ability of the heart to inactivate oxygen metabolites) was significantly lower in old hearts than in younger animals, and that this finding was associated with decreased cardiac concentrations of the scavenger enzymes glutathione peroxidase and $\mathrm{Mn}$ superoxide dismutase. The authors concluded that in rat heart metabolic and functional tolerance toward oxidative stress decreases with age. These observations indicate that the heart undergoes anatomic and functional changes over aging, the interaction of which may eventually result in excessive risk for cardiovascular diseases.

In elderly patients, in addition to age-related changes in function and anatomy of the cardiovascular system, a progression of coronary artery disease and organ damage 
associated with hypertension, diabetes, and often complicated by heart failure are found.

Abnormalities in mitochondrial function, calcium handling, electrolytes alterations, hormones, oxidative stress, and cardioprotective signaling have all been proposed as potentially implicated [6]. Effects of high levels of insulin, involved in many age-related diseases, on the cardiovascular function are well studied, even if conflicting data are reported in literature about the effects of insulin on myocardial contractility [7]. In a model of isolated rats papillary muscles, it was demonstrated that insulin-induced modulation of contractility is calcium independent and that insulin leads to a supersensitization on the betal-adrenoceptors [8], involved in pathogenesis of age-related cardiac diseases, such as heart failure [9]. It has been demonstrated that one of the pathophysiological mechanisms involved in the genesis of heart failure is represented by a persistent beta1adrenoceptors stimulation, that evokes a multitude of cardiac toxic effects, including myocyte apoptosis and hypertrophy, as showed in vivo on rodent hearts and in vitro on cultured cardiomyocytes [10-13].

Regarding the effects of electrolytes changes implicated in the regulation of myocardial function, for instance, it has been demonstrated that magnesium interferes on failed cardiac contractility [14], by modifying sarcoplasmic reticular $\mathrm{Ca}^{2+}$ transport systems with a calcium antagonism mechanism based on competition between $\mathrm{Mg}^{2+}$ and $\mathrm{Ca}^{2+}$ for the same binding sites on key myocardial contractile proteins, such as troponin $\mathrm{C}$, myosin, and actin [15] that could explain the opposite effects of $\mathrm{Mg}^{2+}$ and $\mathrm{Ca}^{2+}$ on myocardial contractility [16].

Furthermore, the aging is characterized by an impaired responsiveness to stress and by a reduced efficiency of endogenous protective mechanisms (i.e., ischemic preconditioning and postconditioning), which results in increased vulnerability to injury $[17,18]$.

A greater myocardial dysfunction induced by ischemiareperfusion injury [19] represents another feature of aging process, then reinforcing the association with oxidative stress. The oxidative stress has been suggested to be also responsible for some metabolic changes.

In particular, old animals show increased release of glutathione and decreased release of oxidized glutathione, suggesting that cardiac oxidative tolerance (considered as the ability of heart to suffer oxidative insults without crucial effects) decreases with age [5]. Ferrara et al. [20] also demonstrated that impairment of systolic and diastolic functions, induction of arrhythmias, release of glutathione, and other abnormalities caused by oxidant exposure can be prevented through antioxidants administration. The oxidative stress that is responsible for these changes, represents an imbalance between production of reactive oxygen species (ROS) (such as nitrogen, superoxide anion, and hydrogen peroxide) and antioxidants cellular systems unable to cope with the excess of oxidants. ROS are continuously formed during life as result of the metabolism of oxygen, and their production is increased during some pathological processes. Mitochondrial respiration is a physiological source of ROS and impairment in this system has been related to dysfunction of several homeostatic processes, such as membrane lipid peroxidation, enzymes inactivation, and nucleic acid bases damage. The protective systems involved in antioxidant cellular defense are principally represented by peroxidase, superoxide dismutases (SOD), and heat shock proteins (HSPs). The SOD catalyze the dismutation of superoxide into oxygen and hydrogen peroxide during physiological and pathological conditions, including aging. It has been demonstrated that the expression and activity of the SOD system are modified in aging, with reduced cell ability to counteract the oxidant molecules, and consequent weak resistance to ROS accumulation [21]. Obviously, cytotypes with limited replication ability, such as brain and heart, are particularly vulnerable to this phenomenon, suggesting that it could explain, at least in part, high prevalence of cardiovascular and neurological disorders in old people [22]. In fact it is widely known that oxidative stress and reduced antioxidant defense have negative effects on cardiac structure and function [23], and they are also involved in lipid membrane oxidation and other heart age-related conditions. Rinaldi et al. [21] showed that high levels of cardiac lipid peroxidation in sedentary old rats were not paired by increased SOD expression. These findings suggested that aging could be associated with increased oxidants without significant changes in antioxidant activity [24]. HSPs are another system of cellular defense against oxidative stress. These "stress-induced proteins" are ubiquitous and highly conserved chaperones, important in the folding of new synthesized or damaged proteins. Moreover HSPs mediate mitochondrial protection against oxidative stress and some of those such as HSP70 have been associated with myocardial protection. Martin et al. [25] showed an increased survival in HSP70-transfected cardiomyocytes and consequent increased expression of the HSP70 enzyme against ischemic cardiac damage. The Rinaldi's study [21] suggested that aging adversely affects HSP70 protein expression with increased levels of HSP27.

Snoeckx et al. [26] showed doubled HSP27 levels during the first 16 hours after heat shock. It is probable that many different aspects of the aged heart are at least partially caused by accumulation of oxidative damage. It has been found that cardiac fibrosis and size of myocytes increase with aging, while the number of myocytes decreases and ventricular hypertrophy is almost a constant finding in the aging rat heart [27-29]. All these findings may be related to oxidative stress, change of calcium homeostasis, with reference to the prolonged calcium transient and contraction, and decreased calcium sequestration by sarcoplasmic reticulum, characteristics of senescent heart. Another important mechanism involved in cellular aging process is represented by family of sirtuins, a cluster of proteins composed by seven homologues that regulate cellular biology and metabolism through deacetylation of histones and other cellular factors such as NFkB, HSF1, p53, FOXOs, and PGC1. SIRT1, the human homologous of the family, is involved in many functions of human physiology, including DNA repair, cell cycle regulation, apoptosis, gene expression, and aging [30]. By FOXO3 acetylation and/or phosphorylation oxidative stress induces arrangement of SIRT1-FOXO3a, 
complex indispensable for cell cycle arrest and induction of DNA repair [31]. SIRT1 can modulate the cellular stress response directly deacetylating some proteins and regulating their expression [32]. In fact SIRT1 inhibition determines transcription suppression of genes activated by exposure to heat shock. On the contrary, SIRT1 activation byagents such as resveratrol, or SIRT1 overexpression enhances the heat shock response [33]. Furthermore, SIRT1 modulates the threshold of cell death in the setting of exogenous stress, including oxidative damage, interacting with p53, inhibits Bax-induced apoptosis by deacetylation of Ku70, and regulation of other targets linked to cell death [34]. Then the ability of SIRT1 to modulate stress resistance is multifaceted and it is not only linked to oxidative stress, but also to other stressful stimuli that play a role in the cellular aging, such as telomere shortening and other types of genotoxic stress.

\section{Physical Activity and Oxidative Stress}

The physical activity increases the expression and the activity of antioxidant enzymes, with consequent reduction of ROS. In part, it seems that positive effects of the physical exercise on senile heart in terms of antioxidant activity could be ascribable to a greater expression and activity of SOD and HSPs.

Rinaldi et al. [21] showed that a physical training program induced high levels of SOD and increased HSP70 and of HSP27 expression in trained old rats compared to sedentary old and young rats. This finding was in accordance with other studies where training protocols were able to increase SOD activity in sedentary old rats [24].

Physical activity has been demonstrated able to reduce generation of oxidants during ischemia-reperfusion damage and to have a calcium-protective role via activation of the ROS scavenger MnSOD. This better oxidative status consequent to a correct program of physical activity is partially responsible for some benefits (such as decreased arterial stiffness, improved endothelial function and metabolic and clotting setting, and reduced body weight).

The relation between aging and accumulation of oxidatively damaged proteins, lipids, and nucleic acids explains how a high resistance to the oxidative stress is associated with increased lifespan, as suggested by many studies in various model organisms $[35,36]$. An 8-week training period has been shown to reduce body weight and cardiac abnormalities characteristic of senescence heart, such as structural changes, ventricular systolic pressure, left ventricle weight/body weight ratio, and heart rate [37], suggesting that exercise training antioxidant effects might be also mediated by mechanisms involving metabolic pathways.

Other findings derived from clinical studies show that regular physical activity decreases cardiovascular comorbidity and mortality in adult and in elderly by restoring the protective effect of ischemic preconditioning [38] and partially contrasting loss of antioxidant defense in the aged heart [37]. In particular, it has been demonstrated that the physical activity in the heart failure works at cardiac level increasing angiogenesis and at levels of adrenal medulla restoring the GRK2-alpha 2 adrenergic receptor axis which regulates the production of catecholamines [39, 40], and therefore it is able to increase norepinephrine release in response to a preconditioning stimulus [41]. This phenomenon has been suggested to be also involved in the cardioprotective effects of exercise training and caloric restriction. In fact, it has been demonstrated that exercise training and food restriction produce together total preservation of ischemic preconditioning in the aging heart, and that one of the mechanisms responsible for early ischemic preconditioning conservation in the aging heart maybe the restoration of norepinephrine release in response to preconditioning stimulus, as confirmed by the absence of the protection by reserpine administration $[42,43]$

Thus physical activity delays the damage accumulation and consequent physiological dysfunction characteristic of aging process.

An interesting observation is that both exercise training and caloric restriction improve the antioxidant system, and this could also explain the analogy between benefits derived by these tools.

By observation that caloric restriction induces increased lifespan by induction of sirtuins activity and that both exercise training and caloric restriction share many benefits it has been hypothesized that SIRT1 is also involved in the physical activity effects, whereas only caloric-restricted subjects showed an increased lifespan [1].

SIRT1 has been implicated in many different processes, such as glucose metabolism and fat mobilization/metabolism. It is possible that SIRT1 mediates skeletal muscle adaptations to endurance exercise by regulating cytosolic $\mathrm{NAD}^{+} / \mathrm{NADH}$ ratio changes during muscular contraction. It has been shown that physical activity is able to increase activity of SIRT1 in aged old rats [22], and this finding could be linked to increased NOS expression in skeletal muscle after contraction induced by electrical stimulation in vivo [44].

\section{Cellular Models of Physical Activity and Aging}

Physical activity is one of the best methods in treatment of age-related chronic-degenerative diseases and in prevention of changes associated with aging.

The selection of exercise program is also important, in fact it has been noticed that antioxidant effects could increase, decrease, or not change in relation with the exercise protocol [37]. Recommendable physical activity should be aerobic, with constant and regular exercise training, not vigorous or intense and planned adjusting the physical activity to the characteristics of subject in order to avoid injuries and to represent useful means of prevention in the elderly. Although the beneficial effects of the exercise are well known in terms of pathophysiology and hemodynamics, very little is known about the molecular bases that are responsible for the effectiveness of physical activity in humans, as previously mentioned. Moreover all studies carried out to investigate this topic have been performed "in vivo" on humans or by 
using “in vitro/in vivo" animal models. In particular, study on human sera is not able to show the cellular changes induced by physical activity, such as in vivo experiments in animal model could not be translated to human experience. A demonstration of the controversial debate on this topic is provided by the recent review by Lee et al. [45] that reported that in-human oxidative stress represents a key feature in all stages of cardiovascular disease. In particular, the authors underline the importance of in-human measurement of oxidative stress circulating biomarkers, some of them with predictive value in cardiovascular disease, but Lee et al. also concluded that these biomarkers do not necessarily reflect intravascular oxidative stress and therefore cannot be used as therapeutic targets or markers to monitor pharmacological treatments in clinical settings.

On the other hand, in animal models several studies demonstrated the molecular basis underlining the physical activity effects. Yang et al. [46] showed that training induces a significant enlargement of collateral vessels in striated ischemic muscle and that these effects were lost upon inhibition of NO production. Eksakulkla et al. [47] suggested that physical activity could improve endothelial dysfunction in aged rats by increasing NO bioavailability. Moreover, Ferrara et al. [37] demonstrated that exercise training, which significantly increases SIRT1 activity, could counteract agerelated systems impairment, by activation of antioxidant systems and DNA repair and disability [48], suggesting therefore a possible role of exercise training in conditioning lifespan (Figure 1).

Although studies in animal models help to examine the changes induced by exercise both at the molecular and functional level, they may not accurately represent what happens in humans in their entirety and complexity during aging.

Obviously, in order to better understand the changes induced by exercise in the elderly, it is necessary to first define "normality" of such modifications, by studying what physical activity induces on blood and cells in healthy young subjects.

An interesting approach is represented by that one used by Conti [49]. Based on Allard's paper [50] in which the authors examined the effects of sera from calorie-restricted subjects on cultured cells, Conti et al. investigated the effects of sera collected from three groups of athletes characterized by different types of sports (aerobic, anaerobic, or mixed) on human endothelial cells [49]. After determination of some antioxidant enzymes activities and levels of oxidants in the sera, they used the same sera to treat cellular lines. In particular human endothelial cell lines EA.hy 926 (ECs) and primary human umbilical vein (HUVEC) were used for the in vitro experiments. The method consisted in growing EA.hy 926 and HUVEC in an endothelial growth medium supplemented with the athletes' sera and exposed or not to oxidative stress. On these cultures the levels of different enzymes and oxidants molecules, and parameters of survival, senescence, and viability were determined. By these experiments the authors demonstrated that survival and proliferation rates were higher in ECs supplemented with serum of athletes performing an aerobic training than in ECs treated with sera of a athletes performing an anaerobic or

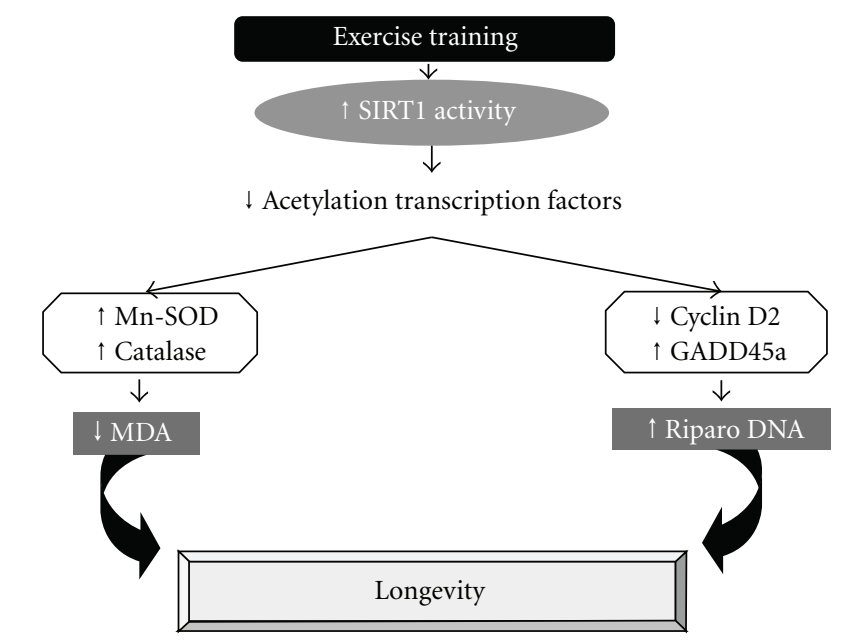

FIGURE 1: Mechanism of exercise training through SIRT1 activity on antioxidant enzymes and factors involved in cell cycle. Exercise training induces increased SIRT1 activity that is responsible both of raised activation of antioxidants system (by MnSOD and Catalase) and cell cycle arrest to promote DNA repair. These phenomena could induce extended lifespan.

mixed sport. Moreover, by the evidence that changes in many functional aspects of cell behavior, including proliferation, are closely related to NO bioavailability $[51,52]$ and that NO production is strongly limited in senescent ECs [53]. In Conti's paper [49] the highest survival and proliferation rates and the lowest senescence observed in endothelial cells conditioned with aerobic serum, both at baseline and after stress induction, suggest that these changes could depend, at least in part, on high NO bioavailability in the aerobic exercise serum. Therefore, the high NO bioavailability in serum from athletes undergoing aerobic training produces favorable effects on human endothelial cells. Finally they concluded that different types of exercise training induced different molecular effects in terms of survival, morphology, and antioxidant system efficiency [49].

The in vivo/vitro technique used in this paper is interesting because for the first time connects the physiological effects of physical activity on the blood cells to molecular changes induced by the same blood cells on endothelial cells, by helping to shed light on the molecular basis of effects of long-term physical activity in humans. The issue of how exercise may favorably affect the modifications related to aging is even more complex. In fact, in aging investigation it is difficult to discern the effects of senescence "per se" from those produced by conditions highly prevalent, such as hypertension, diabetes, and atherosclerosis [54, 55]. Based on these data, that represent the basis to understand the "normal" response of different sera in these "in vitro/vivo" experiments we have planned a similar study.

Cardiac rehabilitation, including physical exercise as an integral part, has been recognized as a fundamental component in the continuum of care for patients with heart failure. Advancements in the understanding of oxidative metabolism, the effects of physical activity on oxidative stress 
[56], the intracellular energy transfer in both skeletal and cardiac muscle, and mechanisms of endothelial dysfunction provide possible explanations of the pathophysiologic factors involved in the development of exercise intolerance in chronic heart failure patients [57], but again they are not sufficient to represent a molecular target for treatment. Exercise training promotes SIRT1 activity [22] in animal models and it improves cardiovascular function and enhances endothelial homeostasis in patients with cardiovascular diseases. However, also the study on aging and heart failure and their connections are addressed only on pathophysiology or cellular aspects, because the limitation in the available tools. By all of the evidence, the elderly with heart failure may represent the population that may derive more benefits from an understanding of these processes. From this point of view the technique used by Conti et al. [49] could represent a good tool to better understand how the exercise training mediates its effects on aging-related diseases, in particular in elderly with heart failure.

\section{References}

[1] G. Corbi, V. Conti, G. Scapagnini, A. Filippelli, and N. Ferrara, "Role of sirtuins, calorie restriction and physical activity in aging," Frontiers in Bioscience (Elite Edition), vol. 4, pp. 768$778,2012$.

[2] S. Vasto, G. Scapagnini, M. Bulati et al., "Biomarkes of aging," Frontiers in Bioscience (Scholar Edition), vol. 2, pp. 392-402, 2010.

[3] M. Galderisi, V. S. Lomoriello, A. Santoro et al., "Differences of myocardial systolic deformation and correlates of diastolic function in competitive rowers and young hypertensives: a speckle-tracking echocardiography study," Journal of the American Society of Echocardiography, vol. 23, no. 11, pp. 1190-1198, 2010.

[4] C. H. Davies, N. Ferrara, and S. E. Harding, “ $\beta$-Adrenoceptor function changes with age of subject in myocytes from nonfailing human ventricle," Cardiovascular Research, vol. 31, no. 1, pp. 152-156, 1996.

[5] P. Abete, C. Napoli, G. Santoro et al., "Age-related decrease in cardiac tolerance to oxidative stress," Journal of Molecular and Cellular Cardiology, vol. 31, no. 1, pp. 227-236, 1999.

[6] S. Besse, C. Delcayre, B. Chevalier et al., "Is the senescent heart overloaded and already failing?" Cardiovascular Drugs and Therapy, vol. 8, no. 4, pp. 581-587, 1994.

[7] G. Vitale, M. Galderisi, A. Colao et al., "Circulating IGF-I levels are associated with increased biventricular contractility in top-level rowers," Clinical Endocrinology (Oxford), vol. 69, no. 2, pp. 231-236, 2008.

[8] N. Ferrara, P. Abete, G. Corbi et al., "Insulin-induced changes in $\beta$-adrenergic response: an experimental study in the isolated rat papillary muscle," American Journal of Hypertension, vol. 18, no. 3, pp. 348-353, 2005.

[9] L. Amato, G. Paolisso, F. Cacciatore et al., "Congestive heart failure predicts the development of non-insulin-dependent diabetes mellitus in the elderly. The Osservatorio Geriatrico Regione Campania Group," Diabetes and Metabolism, vol. 23, no. 3, pp. 213-218, 1997.

[10] M. Zheng, W. Zhu, Q. Han, and R. P. Xiao, "Emerging concepts and therapeutic implications of $\beta$-adrenergic receptor subtype signaling," Pharmacology and Therapeutics, vol. 108, no. 3, pp. 257-268, 2005.
[11] N. Ferrara, K. Davia, P. Abete, F. Rengo, and S. E. Harding, "Alterations in $\beta$-adrenoceptor mechanisms in the aging heart. Relationship with heart failure," Aging-Clinical and Experimental Research, vol. 9, no. 6, pp. 391-403, 1997.

[12] G. Rengo, C. Zincarelli, G. D. Femminella et al., "Myocardial $\beta_{2}$-adrenoceptor gene delivery promotes coordinated cardiac adaptive remodelling and angiogenesis in heart failure," British Journal of Pharmacology, vol. 166, no. 8, pp. 2348-2361, 2012.

[13] G. Rengo, A. Lymperopoulos, C. Zincarelli et al., "Blockade of $\beta$-adrenoceptors restores the GRK2-mediated adrenal $\alpha_{2}$ adrenoceptor-catecholamine production axis in heart failure," British Journal of Pharmacology, vol. 166, no. 8, pp. 2430-2440, 2012.

[14] G. Corbi, D. Acanfora, G. L. Iannuzzi et al., "Hypermagnesemia predicts mortality in elderly with congestive heart disease: relationship with laxative and antacid use," Rejuvenation Research, vol. 11, no. 1, pp. 129-138, 2008.

[15] K. L. Koss and R. D. Grubbs, "Elevated extracellular $\mathrm{Mg}^{2+}$ increases $\mathrm{Mg}^{2+}$ buffering through a Ca-dependent mechanism in cardiomyocytes," American Journal of Physiology, vol. 267, no. 2, pp. C633-C641, 1994.

[16] S. Kawano, "Dual mechanisms of $\mathrm{Mg}^{2+}$ block of ryanodine receptor $\mathrm{Ca}^{2+}$ release channel from cardiac sarcoplasmic reticulum," Receptors and Channels, vol. 5, no. 6, pp. 405-416, 1998.

[17] G. Longobardi, P. Abete, N. Ferrara et al., "'Warm-up' phenomenon in adult and elderly patients with coronary artery disease: further evidence of the loss of "ischemic preconditioning" in the aging heart," Journals of GerontologySeries A, vol. 55, no. 3, pp. M124-M129, 2000.

[18] P. Abete, A. Cioppa, C. Calabrese et al., "Ischemic threshold and myocardial stunning in the aging heart," Experimental Gerontology, vol. 34, no. 7, pp. 875-884, 1999.

[19] C. P. Campobasso, A. S. Dell'Erba, A. Addante, F. Zotti, A. Marzullo, and M. F. Colonna, "Sudden cardiac death and myocardial ischemia indicators: a comparative study of four immunohistochemical markers," American Journal of Forensic Medicine and Pathology, vol. 29, no. 2, pp. 154-161, 2008.

[20] N. Ferrara, P. Abete, G. Ambrosio et al., "Protective role of chronic ubiquinone administration on acute cardiac oxidative stress," Journal of Pharmacology and Experimental Therapeutics, vol. 274, no. 2, pp. 858-865, 1995.

[21] B. Rinaldi, G. Corbi, S. Boccuti et al., "Exercise training affects age-induced changes in SOD and heat shock protein expression in rat heart," Experimental Gerontology, vol. 41, no. 8, pp. 764-770, 2006.

[22] A. Navarro-Arévalo, C. Cañavate, and M. J. Sánchez-delPino, "Myocardial and skeletal muscle aging and changes in oxidative stress in relationship to rigorous exercise training," Mechanisms of Ageing and Development, vol. 108, no. 3, pp. 207-217, 1999.

[23] P. K. Singal, A. Petkau, J. M. Gerrard, S. Hrushovetz, and J. Foerster, "Free radicals in health and disease," Molecular and Cellular Biochemistry, vol. 84, no. 2, pp. 121-122, 1988.

[24] F. Gündüz, U. K. Sentürk, O. Kuru, B. Aktekin, and M. R. Aktekin, "The effect of one year's swimming exercise on oxidant stress and antioxidant capacity in aged rats," Physiological Research, vol. 53, no. 2, pp. 171-176, 2004.

[25] J. L. Martin, R. Mestril, R. Hilal-Dandan, L. L. Brunton, and W. H. Dillmann, "Small heat shock proteins and protection against ischemic injury in cardiac myocytes," Circulation, vol. 96, no. 12, pp. 4343-4348, 1997.

[26] L. H. E. H. Snoeckx, R. N. Cornelussen, F. A. Van Nieuwenhoven, R. S. Reneman, and G. J. Van Der Vusse, "Heat shock 
proteins and cardiovascular pathophysiology," Physiological Reviews, vol. 81, no. 4, pp. 1461-1497, 2001.

[27] P. Anversa, B. Hiler, R. Ricci, G. Guideri, and G. Olivetti, "Myocyte cell loss and myocyte hypertrophy in the aging rat heart," Journal of the American College of Cardiology, vol. 8, no. 6, pp. 1441-1448, 1986.

[28] S. Besse, V. Robert, P. Assayag, C. Delcayre, and B. Swynghedauw, "Nonsynchronous changes in myocardial collagen mRNA and protein during aging: effect of DOCA-salt hypertension," American Journal of Physiology, vol. 267, no. 6, pp. H2237-H2244, 1994.

[29] M. Klima, T. R. Burns, and A. Chopra, "Mycardial fibrosis in the elderly," Archives of Pathology and Laboratory Medicine, vol. 114, no. 9, pp. 938-942, 1990.

[30] O. Grubisha, B. C. Smith, and J. M. Denu, "Small molecule regulation of Sir2 protein deacetylases," FEBS Journal, vol. 272, no. 18, pp. 4607-4616, 2005.

[31] A. Brunet, L. B. Sweeney, J. F. Sturgill et al., "Stress-dependent regulation of FOXO transcription factors by the SIRT1 deacetylase," Science, vol. 303, no. 5666, pp. 2011-2015, 2004.

[32] M. Porcu and A. Chiarugi, "The emerging therapeutic potential of sirtuin-interacting drugs: from cell death to lifespan extension," Trends in Pharmacological Sciences, vol. 26, no. 2, pp. 94-103, 2005.

[33] S. D. Westerheide, J. Anckar, S. M. Stevens Jr., L. Sistonen, and R. I. Morimoto, "Stress-inducible regulation of heat shock factor 1 by the deacetylase SIRT," Science, vol. 323, no. 5917, pp. 1063-1066, 2009.

[34] H. Y. Cohen, C. Miller, K. J. Bitterman et al., "Calorie restriction promotes mammalian cell survival by inducing the SIRT1 deacetylase," Science, vol. 305, no. 5682, pp. 390-392, 2004.

[35] Z. Ungvari, R. Buffenstein, S. N. Austad, A. Podlutsky, G. Kaley, and A. Csiszar, "Oxidative stress in vascular senescence: lessons from successfully aging species," Frontiers in Bioscience, vol. 13, no. 13, pp. 5056-5070, 2008.

[36] Heart Protection Study Collaborative Group, "MRC/BHF Heart Protection Study of antioxidant vitamin supplementation in 20536 high-risk individuals: a randomised placebocontrolled trial," The Lancet, vol. 360, no. 9326, pp. 23-33, 2002.

[37] N. Ferrara, B. Rinaldi, G. Corbi et al., "Exercise training promotes SIRT1 activity in aged rats," Rejuvenation Research, vol. 11, no. 1, pp. 139-150, 2008.

[38] P. Abete, N. Ferrara, F. Cacciatore et al., "High level of physical activity preserves the cardioprotective effect of preinfarction angina in elderly patients," Journal of the American College of Cardiology, vol. 38, no. 5, pp. 1357-1365, 2001.

[39] D. Leosco, G. Rengo, G. Iaccarino et al., "Exercise promotes angiogenesis and improves $\beta$-adrenergic receptor signalling in the post-ischaemic failing rat heart," Cardiovascular Research, vol. 78, no. 2, pp. 385-394, 2008.

[40] G. Rengo, D. Leosco, C. Zincarelli et al., "Adrenal GRK2 lowering is an underlying mechanism for the beneficial sympathetic effects of exercise training in heart failure," American Journal of Physiology-Heart and Circulatory Physiology, vol. 298, no. 6, pp. H2032-H2038, 2010.

[41] P. Abete, C. Calabrese, N. Ferrara et al., "Exercise training restores ischemic preconditioning in the aging heart," Journal of the American College of Cardiology, vol. 36, no. 2, pp. 643$650,2000$.

[42] P. Abete, G. Testa, G. Galizia et al., "Tandem action of exercise training and food restriction completely preserves ischemic preconditioning in the aging heart," Experimental Gerontology, vol. 40, no. 1-2, pp. 43-50, 2005.

[43] P. Abete, G. Testa, N. Ferrara et al., "Cardioprotective effect of ischemic preconditioning is preserved in food-restricted senescent rats," American Journal of Physiology, vol. 282, no. 6, pp. H1978-H1987, 2002.

[44] E. Nisoli, C. Tonello, L. Briscini, and M. O. Carruba, "Inducible nitric oxide synthase in rat brown adipocytes: implications for blood flow to brown adipose tissue," Endocrinology, vol. 138, no. 2, pp. 676-682, 1997.

[45] R. Lee, M. Margaritis, K. M. Channon, and C. Antoniades, "Evaluating oxidative stress in human cardiovascular disease: methodological aspects and considerations," Current Medicinal Chemistry, vol. 19, no. 16, pp. 2504-2520, 2012.

[46] H. T. Yang, B. M. Prior, P. G. Lloyd et al., "Training-induced vascular adaptations to ischemic muscle," Journal of Physiology and Pharmacology, vol. 59, no. 7, pp. 57-70, 2008.

[47] S. Eksakulkla, D. Suksom, P. Siriviriyakul, and S. Patumraj, "Increased NO bioavailability in aging male rats by genistein and exercise training: using 4, 5-diaminofluorescein diacetate," Reproductive Biology and Endocrinology, vol. 7, article 93, 2009.

[48] F. Cacciatore, P. Abete, S. Maggi et al., "Disability and 6-year mortality in elderly population. Role of visual impairment," Aging-Clinical and Experimental Research, vol. 16, no. 5, pp. 382-388, 2004.

[49] V. Conti, G. Corbi, G. Russomanno et al., "Oxidative stress effects on endothelial cells treated with different athletes' sera," Medicine \& Science in Sports \& Exercise, vol. 44, no. 1, pp. 3949, 2012.

[50] J. S. Allard, L. K. Heilbronn, C. Smith et al., "In vitro cellular adaptations of indicators of longevity in response to treatment with serum collected from humans on calorie restricted diets," PLoS ONE, vol. 3, no. 9, Article ID e3211, 2008.

[51] A. Hassid, J. Yao, and S. Huang, "NO alters cell shape and motility in aortic smooth muscle cells via protein tyrosine phosphatase 1B activation," American Journal of Physiology, vol. 277, no. 3, pp. H1014-H1026, 1999.

[52] F. Fazio, L. Lionetto, G. Molinaro et al., "Cinnabarinic acid, an endogenous metabolite of the kynurenine pathway, activates type 4 metabotropic glutamate receptors," Molecular Pharmacology, vol. 81, no. 5, pp. 643-656, 2012.

[53] I. Sato, I. Morita, K. Kaji, M. Ikeda, M. Nagao, and S. Murota, "Reduction of nitric oxide producing activity associated with in vitro aging in cultured human umbilical vein endothelial cell," Biochemical and Biophysical Research Communications, vol. 195, no. 2, pp. 1070-1076, 1993.

[54] D. F. Dai and P. S. Rabinovitch, "Cardiac aging in mice and humans: the role of mitochondrial oxidative stress," Trends in Cardiovascular Medicine, vol. 19, no. 7, pp. 213-220, 2009.

[55] D. Dutta, R. Calvani, R. Bernabei, C. Leeuwenburgh, and E. Marzetti, "Contribution of impaired mitochondrial autophagy to cardiac aging: mechanisms and therapeutic opportunities," Circulation Research, vol. 110, no. 8, pp. 1125$1138,2012$.

[56] Z. Ungvári, S. A. Gupte, F. A. Recchia, S. Bátkai, and P. Pacher, "Role of oxidative-nitrosative stress and downstream pathways in various forms of cardiomyopathy and heart failure," Current Vascular Pharmacology, vol. 3, no. 3, pp. 221-229, 2005.

[57] J. Downing and G. J. Balady, "The role of exercise training in heart failure," Journal of the American College of Cardiology, vol. 58, no. 6, pp. 561-569, 2011. 


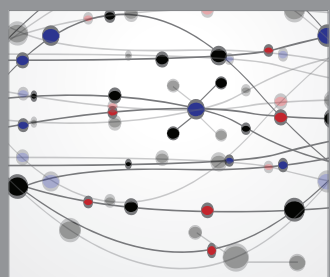

The Scientific World Journal
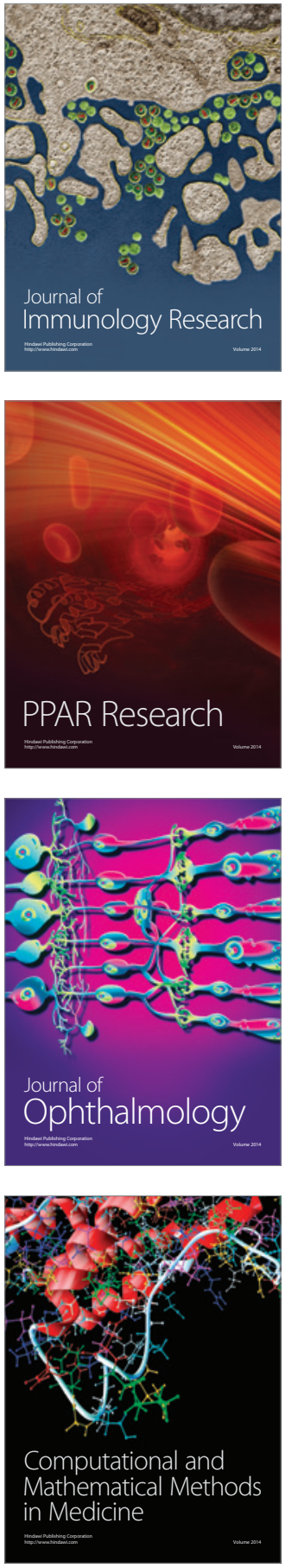

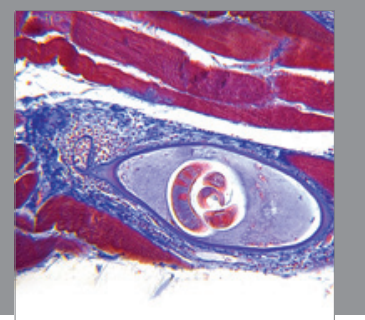

Gastroenterology

Research and Practice
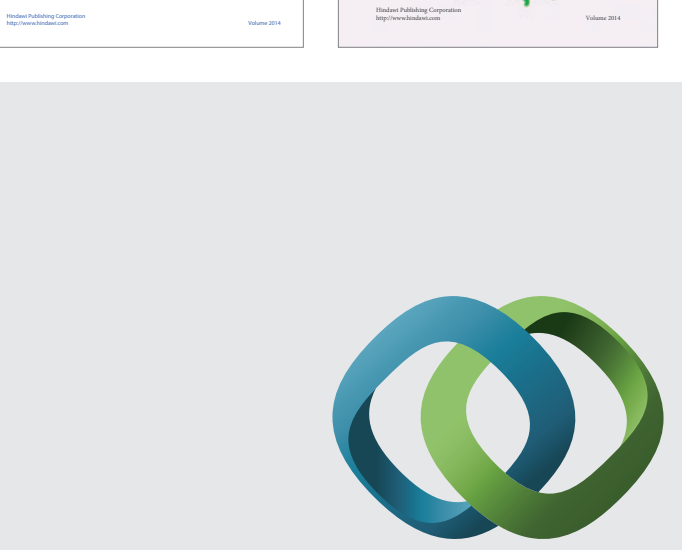

\section{Hindawi}

Submit your manuscripts at

http://www.hindawi.com
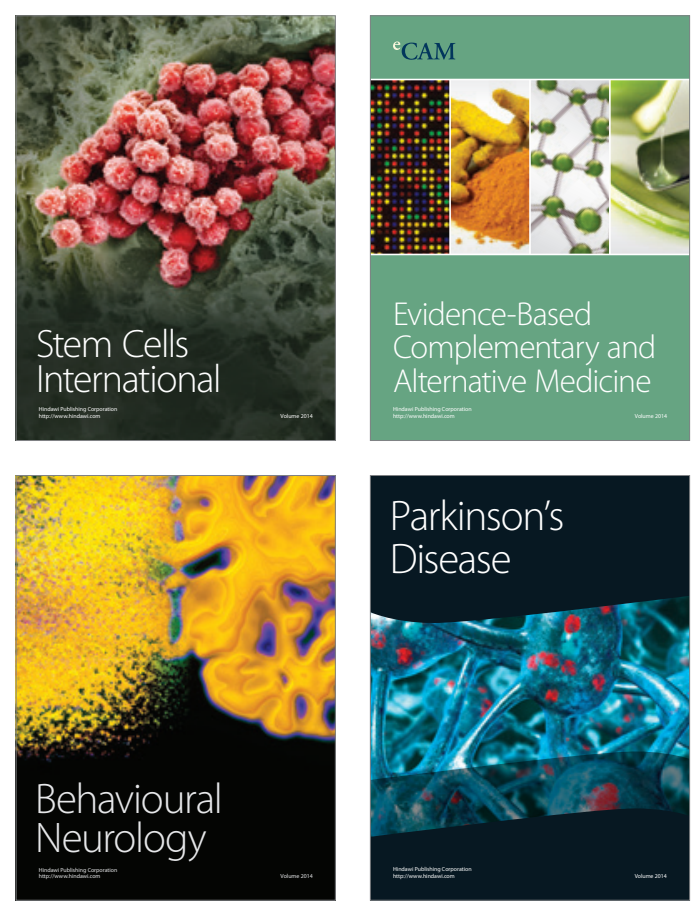

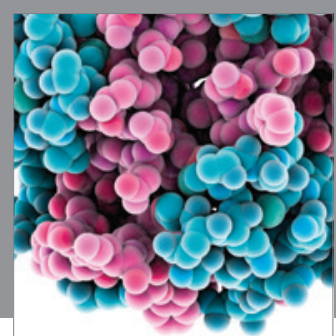

Journal of
Diabetes Research

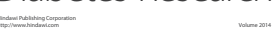

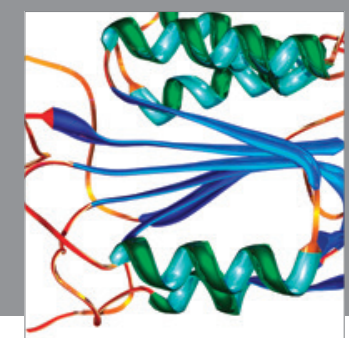

Disease Markers
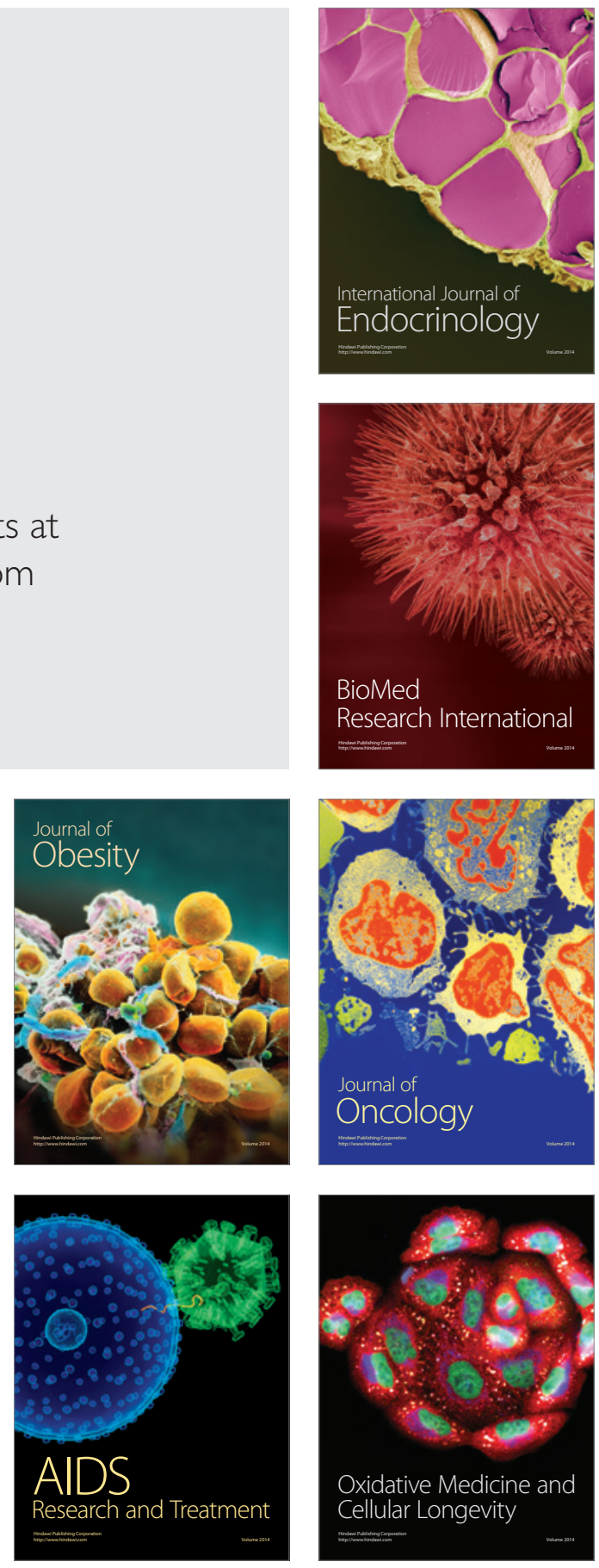